Baltic Astronomy, vol.4, 396-401, 1995.

\title{
PERIOD STABILITY IN AM CVn
}

\section{J. L. Provencal}

Department of Physics, University of Delaware, Newark, DE 19716, U.S.A.

Received September 1, 1995.

\begin{abstract}
The unusual variable star AM CVn has puzzled astronomers for over 40 years. This object, both a photometric and spectroscopic variable, is believed to contain a pair of hydrogen deficient white dwarfs of extreme mass ratio, transferring material via an accretion disk. We have examined the phase stability of the dominant photometric variation at $1902.5 \mu \mathrm{Hz}$, analyzing 289 hours of high speed photometric data spanning 1976 to 1992 , and have determined the dominant frequency to be $(1902.509802 \pm 0.00001) \mu \mathrm{Hz}$, with $\dot{P}=(+1.71 \pm 0.04) \times 10^{-11} \mathrm{~s} \mathrm{~s}^{-1}$.
\end{abstract}

Key words: stars: white dwarfs - stars: oscillations - stars: individual: $\mathrm{AM} C \mathrm{CV}$

\section{Introduction}

AM CVn (HZ 29, WD 1232+37, PG 1232+379) was discovered by Humason \& Zwicky (1947). Its peculiar optical spectrum is characterized by broad, shallow, asymmetric absorption lines of neutral helium and a complete lack of hydrogen (Greenstein \& Matthews 1957). AM CVn is also a photometric variable, varying its brightness at the $1 \%$ level at two independent frequencies; a previously reported double humped $951.3 \mu \mathrm{Hz}(1051.2 \mathrm{~s}$ ) variation (Smak 1967) and $988.7 \mu \mathrm{Hz}(1011.4 \mathrm{~s}$ ) (Solheim et al. 1984). Flickering, a classic signature of mass transfer, is also reported in AM CVn's light curve (Warner \& Robinson 1972). No large magnitude outbursts have been observed.

AM CVn is the prototype interacting binary white dwarf system (IBWDs), believed to contain two helium white dwarfs of extreme 
mass ratio, transferring material via an accretion disk. If we can understand the mechanics behind these objects, we will have a sensitive probe to explore nucleosynthesis, binary star evolution and stellar structure. However, fundamental questions persist. Only circumstantial evidence supports AM CVn's binary nature, we do not see the secondary. The orbital frequency is believed to be $951.3 \mu \mathrm{Hz}$, but all attempts to find radial velocity variations or photometric power at this frequency have failed (Provencal et al. 1995). Despite the $951.3 \mu \mathrm{Hz}$ variation's presumed origin, a predictive ephemeris has not yet been presented. Solheim et al. (1984) first reported significant power at $988.7 \mu \mathrm{Hz}$. If $951.3 \mu \mathrm{Hz}$ is the orbital frequency, we require a second mechanism to produce the $988.7 \mu \mathrm{Hz}$ variation. Finally, Patterson, Halpern \& Shambrook (1993) find line profile variations with a frequency of $20.7 \mu \mathrm{Hz}$ in AM CVn's optical spectra, which they show cannot be the orbital frequency and they interpret as the precession of an elliptical disk.

We consider a thorough examination of the phase stability of AM CVn's photometric variations to be extremely important. We report on our analysis of 289 hours of high speed photometric data spanning 1976 to 1992 . We determine the dominant frequency in the Fourier transform (FT) to be $(1902.509802 \pm 0.00001) \mu \mathrm{Hz}$, with $\dot{P}=(+1.71 \pm 0.04) \times 10^{-11} \mathrm{~s} \mathrm{~s}^{-1}$.

\section{Phase stability}

The traditional method of phase analysis is the $O-C$ diagram, a series of points characterizing a variation's behavior relative to an ephemeris (Kepler et al. 1991). An accurate model for a stable frequency will produce a set of $O-C$ points best fitted by a straight line with zero slope.

Previous $O-C$ calculations for AM CVn, examining the supposed $951.3 \mu \mathrm{Hz}$ variation, use timings derived from a single night of observations, with varying results (see Patterson et al. 1979, Solheim et al. 1984 and Patterson et al. 1992 for examples). Our FTs do not show power at $951.3 \mu \mathrm{Hz}$. The dominant photometric period is actually a doublet, at $1902.5 \mu \mathrm{Hz}$. We have shown (Provencal et al. 1995) that the $1902.5 \mu \mathrm{Hz}$ doublet requires 13.4 hours of continuous data to resolve, so a timing from a single night of observations is not accurate. If the FTs do not contain a sufficient time base to resolve the web of aliases, the aliases from the small component contribute significantly to the power in the dominant peak. 
We will minimize these problems by calculating seasonal timings for the $1902.5 \mu \mathrm{Hz}$ frequency to ensure complete resolution of the power spectra. Provencal et al. (1995) contains a detailed account of the frequency and amplitude stability of this variation, eliminating the possibility of two or more unresolved variations beating together. We determine the dominant frequency for our entire data set to be $(1902.509804 \pm 0.000003) \mu \mathrm{Hz}$.

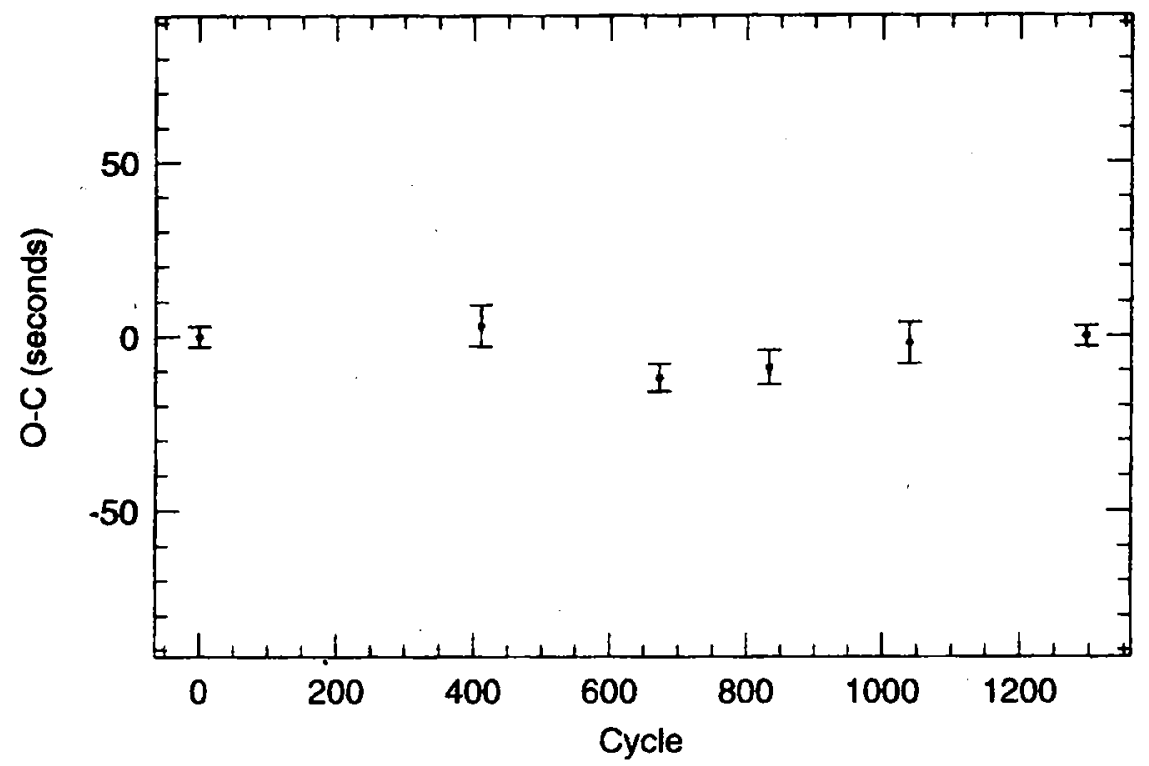

Fig. 1. $O-C$ diagram for the 1990 WET observing run.

We begin by placing a limit on the $1902.5 \mu \mathrm{Hz}$ variation's rate of period change, the details of which are outlined in Provencal et al. (1995). Fig. 1 is the $O-C$ diagram for the best period from the 1990 WET data set, which places a constraint of $\dot{P}<(2.3 \pm 1.4) \times$ $10^{-8} \mathrm{~s} \mathrm{~s}^{-1}$ and eliminates the possibility of random phase changes on timescales of days. This constraint is improved by examining the change in the best frequencies for each season (see Table 2 in Provencal et al. 1995). Using the 1990 and 1992 frequencies, $|\dot{P}|<$ $2 \times 10^{-10} \mathrm{~s} \mathrm{~s}^{-1}$, otherwise we would see a greater change in seasonal frequencies. This figure is consistent with the difference between any two seasons and is comfortably below our earlier limit.

To calculate our $O-C$ points, we fitted a $1902.509804 \mu \mathrm{Hz}$ sinusoid to each observing season's data. Because the 1992 season spans the longest time base, we designate this season's $T_{\max }$ as $T_{\text {zero }}$, 


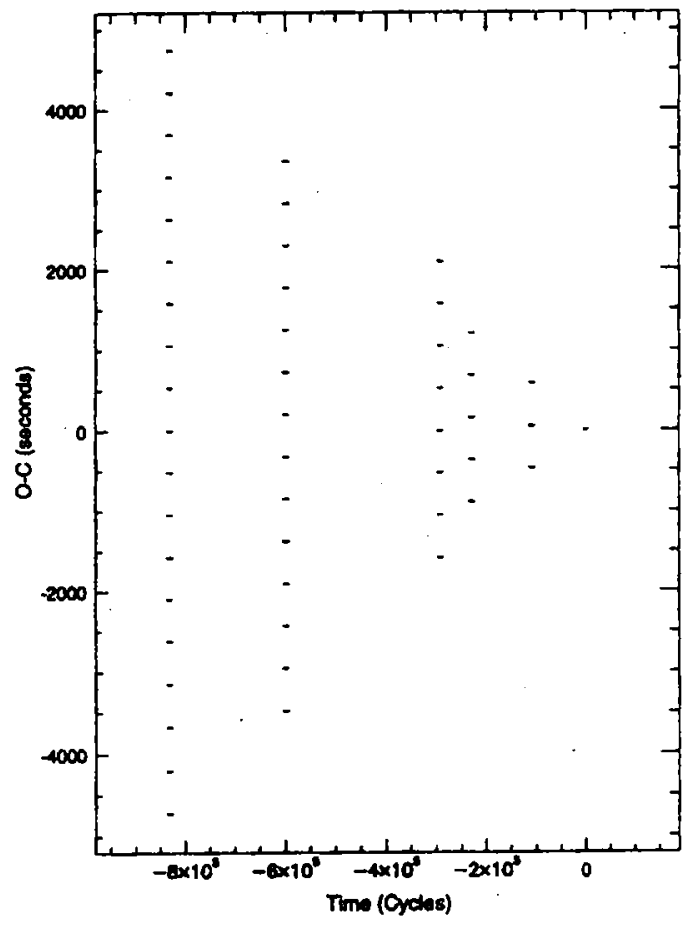

Fig. 2. $O-C$ diagram for AM CVn's $1902.5 \mu \mathrm{Hz}$ variation, representing all possible timings given our limit on the rate of period change. A solution must pass through one timing from each season.

and give it an $O-C$ value of $0 \mathrm{~s}$, and refer other $O-C$ points to this initial timing. Our constrained, but nonzero value of $\dot{P}$ introduces cycle count aliases between observing seasons, greatly increasing the possible solutions to the $O-C$ diagram. From 1990 to 1992 , we could accumulate \pm 1 cycles, eliminating solutions that in 1990 pass through cycle count aliases greater than this. Applying similar limits to other $O-C$ points results in the fan-shaped plot in Fig. 2. In order to prove that we measure a period change, all possible solutions except one must be eliminated to a high degree of confidence.

We calculated fits for values of constant $\dot{P}$ by fitting (Kepler et al. 1991)

$$
(O-C)=T_{\max }^{\mathrm{obs}}-T_{\text {zero }}-P E-\frac{1}{2} P \dot{P} E^{2}
$$




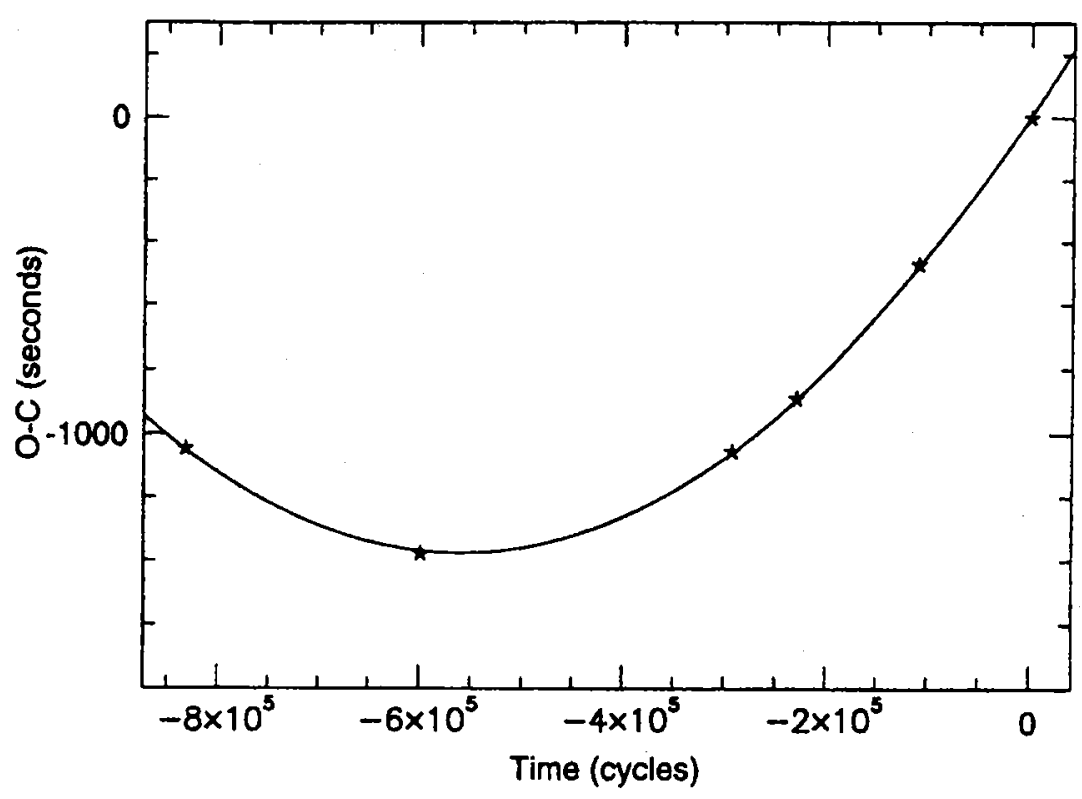

Fig. 3. Best fit to the $O-C$ points in Fig. 2. The $1 \sigma$ errors are the size of the points.

where $E$ is the number of cycles which elapse until $T_{\text {zero }}$ and $P=$ $\frac{1}{f}=525.621470 \mathrm{~s}$, to the points in the $O-C$ diagram. Provencal et al. (1995) outlines the extensive steps we employed. The only fit exhibiting predictive power is $\dot{P}=(+1.68 \pm 0.03) \times 10^{-11} \mathrm{~s} \mathrm{~s}^{-1}$ (Fig. 3). The probability of obtaining 6 random points that lie on a parabola is 1 in 125000 .

To confirm this value for the period change, we fitted a sinusoid with various values of $\dot{P}$ to the entire data set. The best value for $\dot{P}$ obtained via this method is $(+1.73 \pm 0.03) \times 10^{-11} \mathrm{~s} \mathrm{~s}^{-1}$, in agreement with that obtained directly from the $O-C$ diagram. Our measured rate of period change is therefore $(+1.71 \pm 0.04) \times 10^{-11} \mathrm{~s} \mathrm{~s}^{-1}$, corresponding to a timescale of

$$
\frac{P}{\dot{P}} \approx 1 \times 10^{6} \mathrm{yr}
$$

With such a long timescale, it is doubtful that this variation arises in a disk. 


\section{References}

Kepler S.O. et al. 1991, in Proc. of the 7th European Workshop on White Dwarfs (NATO ASI Ser.), eds. G. Vauclair \& E.M. Sion, Kluwer Academic Publishers, p. 143

Patterson J., Nather R.E., Robinson E.L. 1979, ApJ, 232, 819

Patterson J., Sterner E., Halpern J.P., Raymond J.C. 1992, ApJ, 384, 234 Provencal J.L. et al. 1995, ApJ, 445, 927

Solheim J.E., Robinson E.L., Nather R.E., Kepler S.O. 1984, A\&A, 135, 1 